\title{
Azione favorente l'espulsione del calcolo renale da parte dell'acqua oligominerale Rocchetta
}

\author{
N. Di Paolo, L. Capotondo, A. Bruci \\ UO Nefrologia e Dialisi, Azienda Ospedaliera Senese, Siena
}

a medicina ha visto da sempre nella diuresi forzata la miglior profilassi e molto sovente la miglior terapia della calcolosi renale e di ciò fa testimonianza il successo millenario della terapia termale, ma è anche vero che non è mai stata data una spiegazione razionale del fenomeno, né tanto meno si è mai riusciti a capire perché alcune acque funzionino meglio di altre.

Chiunque si interessi di acque minerali ha sempre incontrato enormi, ma ben intuibili, difficoltà ad un approccio medico del problema.

Personalmente ritengo che l'atteggiamento corretto del ricercatore sia quello di valutare non solo l'aspetto biologico della questione, ma di considerare con oculatezza anche la fisica e la chimica dell'acqua per tentare prima di comprendere e poi stendere un razionale di ricerca completo.

\section{Fisica dell'acqua e calcolosi renale}

È opinione comune che la diuresi forzata faciliti per un non meglio identificato "effetto wash-out" la detersione delle vie escretrici urinarie favorendo, da una parte, l'eliminazione di sostanze ivi depositate e dall'altra ostacolando la loro deposizione.

Ma se andiamo ad analizzare la questione più da vicino, possiamo renderci conto che non è poi così semplice.

L'uomo infatti ha una diuresi media di 800-1000 mlldie che corrisponde ad un volume di $0.55-0.69 \mathrm{ml} \backslash \mathrm{mi}-$ nuto che, se considerato su due ureteri, ci darà giusto un effetto di transito di tipo capillare. Se però aumentiamo la diuresi di tre volte (2400 - 3000 mlldie) otterremo un volume minuto di $1.66-2.08 \mathrm{ml}$. Se poi l'introduzione dei liquidi, come avviene solitamente alle terme, si effettua in sole due ore si potrà ottenere, nelle immediate ore successive, una diuresi, a rene funzionante, che potrà arrivare a valori di 25 mllmin, quantità discreta anche se divisa per i due ureteri.

$\mathrm{E}$ indispensabile a questo punto introdurre il concetto di forza di trasporto e di corrosione: la fisica insegna che la quantità di peso unitario di materiali che l'acqua è in grado di trasportare varia in relazione alla portata del corso stesso secondo la formula $\mathrm{P}=\mathrm{Sv}^{6}$ dove $\mathrm{P}$ è la potenza, $\mathrm{S}$ la sezione liquida e $\mathrm{v}$ la sua velocità: se questo concetto sembra ovvio, più sorprendente è sapere che, in base alla formula precedente, se si raddoppia il valore della portata, la quantità dei materiali solidi trasportati da quello stesso corso d'acqua sarà di ben sessantaquattro volte maggiore (1). Questa "legge della sesta potenza" è stata postulata da W. Hopkins nel 1842. Da allora è stata sempre accettata (2), anche se qualcuno ha pensato che la potenza dovrebbe essere la quarta o la quinta e non la sesta (3). In ogni caso l'aumento di tre volte della portata, aumenterà spaventosamente la forza di trasporto e di erosione: se poi teniamo conto del fattore di moltiplicazione che si ha quando il soggetto calcolotico beve in tempi rapidi $2-3$ litri di acqua aumentando la sua diuresi minuto di $30-40$ volte, i numeri diventano davvero grandi.

Ma la parte fisica non finisce qui. Infatti sappiamo che il potere erosivo delle acque è direttamente pro- 
porzionale al materiale stesso che lo scorrimento dell'acqua è capace di rimuovere. In altre parole più l'acqua è ricca di detriti in generale più capacità avrà di staccare e trasportare a valle massi di notevoli dimensioni. I fiumi melmosi in piena hanno capacità di trasporto eccezionali. Tutto ciò è pressoché impossibile da riportare in formule matematiche (3), ma quello che a noi interessa è il fatto paradossale che, se le leggi naturali valgono anche per gli ureteri (ipotesi molto probabile in quanto tali leggi valgono sia per i fiumi sotterranei che per le condutture), più le vie escretrici saranno "sporche" di fini detriti calcarei o organici, più efficace dovrebbe essere il trattamento idroterapico.

Ma il modello dell'uretere umano è più complicato del letto di un ruscello. Infatti per verificare se questi fenomeni avvengono anche nell'uomo, non possiamo fare a meno di creare in un modello sperimentalc anche la peristalsi ureterale.

Studi di fisiologia delle vie escretrici (4) hanno dimostrato che il carico idrico comporta a livello ureterale un aumento vivace della peristalsi ureterale, sia come numero di contrazioni che come durata, (probabile presenza di osmocettori e (o) pressocettori a livello del bacinetto) portando la pressione intraureterale media da valori normali di $5-15 \mathrm{~cm}$ di $\mathrm{H}_{2} \mathrm{O}$ a $50-80 \mathrm{~cm}$ di $\mathrm{H}_{2} \mathrm{O}$. Da parte sua quindi la peristalsi ureterale, aumentata dal carico idrico del bacinetto, non potrà far altro che incrementare ulteriormente la forza di trasporto ed erosione.

Per comprendere meglio il tutto, potremmo portare come esempio un soggetto che abbia della renella depositata sul fondo di qualche calice. Da quello che abbiamo detto è facile intuire come la terapia idropinica con la sua "moltiplicazione di forza" riuscirà abbastanza agevolmente a spostare i sedimenti presenti nelle vie urinarie (infatti sono piuttosto frequenti le emissioni di renella, durante la terapia termale): ma allorché sarà ricco di materiali, il flusso avrà ancora più potenza di trasporto e maggior probabilità di detergere quei detriti più adesi alla mucosa delle vie urinarie e quindi di conseguenza avrà più possibilità di staccare e trasportare i calcoli.

Il problema dell'adesività di un calcolo alla parete delle vie urinarie è quanto mai importante: infatti l'esperienza chirurgica insegna che in genere i calcoli renali sono in parte adesi alla mucosa tramite una matrice organica formata da collagene e fibrina ed altre sostanze proteiche. $\grave{E}$ probabile che le acque possano agire su tale matrice.

Tutto ciò ha bisogno naturalmente di verifica sperimentale, ma ritengo che il razionale della ricerca sia quanto meno corretto.

\section{Chimica dell'acqua e calcolosi}

A mio parere la difficoltà principale di definire un' acqua minerale, nasce dallo stesso concetto attuale di acqua, elemento, fra tutti gli elementi presenti in natura, scientificamente insolito: infatti essa bolle, congela, evapora, galleggia, assorbe e distribuisce calore, differentemente da tutti gli altri composti chimici strutturalmente simili e presenti in natura: infatti se l'acqua dovesse comportarsi come tutte le altre molecole in linea di principio similari $\left(\mathrm{H}_{2} \mathrm{~S}\right.$, $\mathrm{H}_{2} \mathrm{Se}, \mathrm{H}_{2} \mathrm{Te}$ ecc) dovrebbe fondere a $-100^{\circ} \mathrm{C}$ e bollire a $80^{\circ} \mathrm{C}$, congelare a temperature bassissime e assolutamente non galleggiare. L'acqua ha quindi molta più coesione interna di quello che ci si potrebbe aspettare, quasi che tutte queste sue particolari proprietà abbiano il fine specifico di mantenere la vita sulla terra.

L'acqua che nel nostro pianeta è presente contemporaneamente allo stato solido, liquido e gassoso non è poi di così semplice composizione, come al primo approccio potrebbe sembrare; infatti è costituita, allorché si trovi allo stato puro, da ben trentatrè diverse sostanze di cui 18 isotopi e 15 generi di ioni (1) e le sue proprietà fisico-chimiche sono ben lungi dall'essere state totalmente scoperte o chiarite.
Fra i tanti attributi chimici dell'acqua, uno che credo ci interessi molto da vicino, è quello di essere il più potente solvente che si conosca in natura. Infatti la maggior parte delle sostanze sono solubili in acqua: questa ha la forza di sciogliere ed intaccare qualsiasi cosa.

Ciò è tanto vero che almeno la metà degli elementi chimici noti si possono trovare disciolti nelle acque naturali, molti dei quali in grandi quantità, mentre altri solamente in tracce: in pratica mentre ogni lago, corso d'acqua o acqua minerale rappresenta una vera e propria soluzione, il mare e l'acqua corporea sono soluzioni acquose discretamente concentrate che contengono letteralmente migliaia di sostanze allo stato di ioni, fra cui metalli e metalloidi, composti organici ed inorganici.

L'acqua è inoltre un solvente "inerte" nel senso che non viene alterata chimicamente dalla stragrande maggioranza di sostanze che scioglie, ed ha una tensione superficiale più elevata rispetto a qualunque liquido di comune impiego. Da non scordare poi la sua forte capacità di coesione. Queste proprietà ampliano in modo vasto il potere di erosione dell'acqua che è poi proporzionale al suo movimento e quindi alla sua portata. Un altro aspetto importante, come per la fisica così anche per la chimica è ciò che si trova in soluzione nelle acque in generale: nelle sorgive, e nel nostro caso nelle acque minerali, diventa molto importante ciò che ci si ritrova disciolto allorché sgorgano dal sottosuolo.

Un esempio che trovo calzante è quello delle acque minerali di Epson, in Gran Bretagna, dove la composizione chimica dell'acqua è molto particolare; ricchissima in solfato di magnesio essa viene utilizzata come purgante: in questo caso 1 ' effetto terapeutico è eclatante $\mathrm{e}$ la spiegazione del fenomeno è semplice.

Assai più complesso è capire perché alcune acque minerali sono più efficienti di altre acque nella terapia e nella prevenzione della calcolosi urinaria e se ciò dipenda dalla loro composizione chimica. 

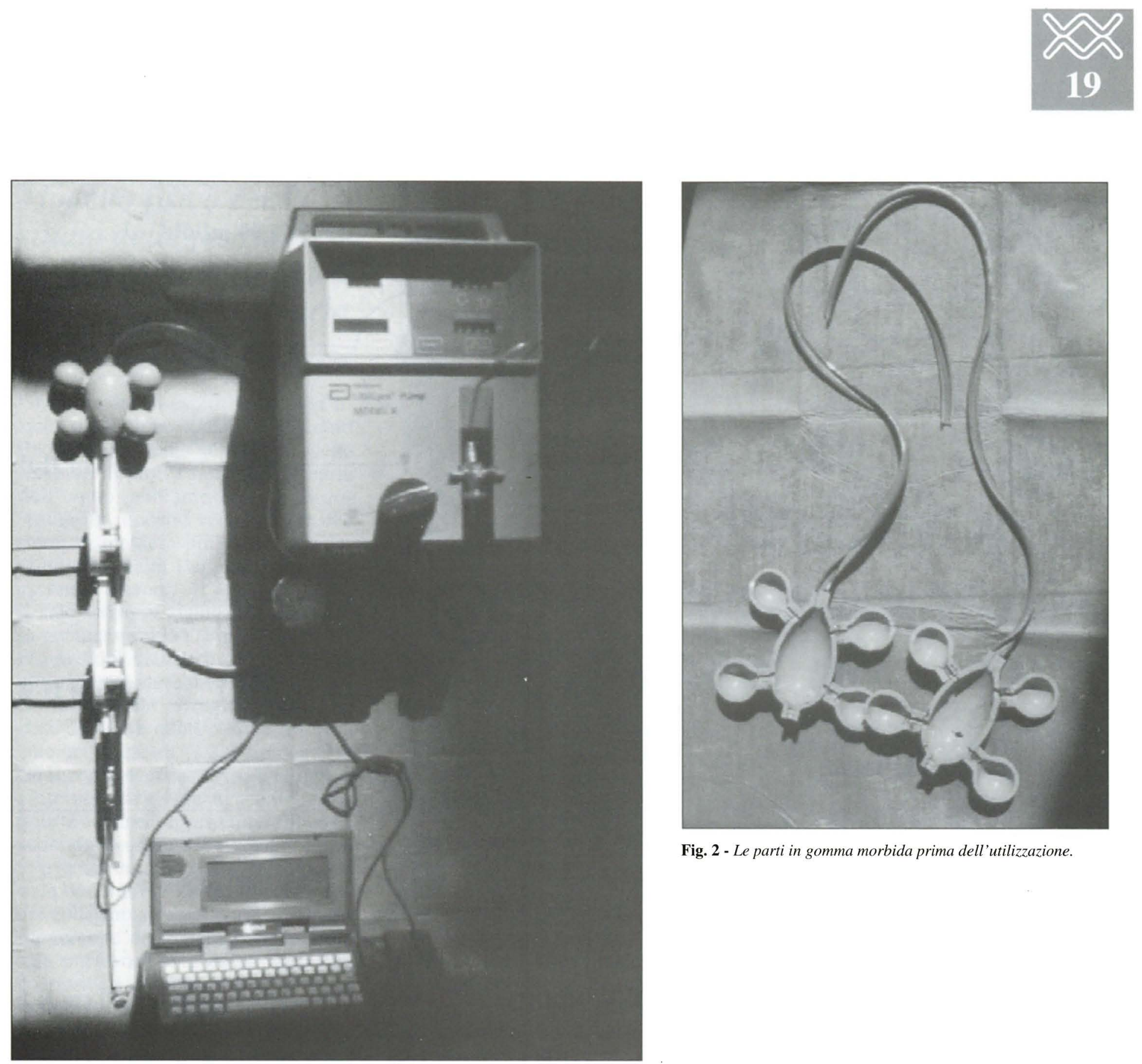

Fig. 2 - Le parti in gomma morbida prima dell'utilizzazione.

Fig. 1 - Il nostro modello in azione.

TABELLA I - STUDIO DELLE PRESSIONI PELVICHE ED URETERALI OTTENUTE CON IL MODELLO SPERIMENTALE

\begin{tabular}{lccc}
\hline $\begin{array}{l}\text { Entrata } \\
\mathrm{ml} / \mathrm{min}\end{array}$ & $\begin{array}{c}\text { Pressione } \\
\text { bacinetto }\end{array}$ & $\begin{array}{c}\text { Pressione } \\
\text { uretere prossimale }\end{array}$ & $\begin{array}{c}\text { Pressione } \\
\text { uretere distale }\end{array}$ \\
\hline 0.55 & $5.27 \pm 0.27$ & $5.16 \pm 0.41$ & $5.40 \pm 0.36$ \\
0.70 & $5.24 \pm 0.25$ & $5.50 \pm 0.33$ & $5.34 \pm 0.44$ \\
1.38 & $5.67 \pm 0.48$ & $5.72 \pm 0.36$ & $7.20 \pm 0.74$ \\
2.08 & $6.18 \pm 0.36$ & $6.21 \pm 1.04$ & $10.12 \pm 1.38^{*}$ \\
33 & $21.73 \pm 7.40 *$ & $22.15 \pm 6.84 *$ & $24.20 \pm 8.10^{*}$ \\
50 & $32.26 \pm 7.14 *$ & $34.67 \pm 8.15 *$ & $38.75 \pm 9.34^{*}$ \\
33 (con p.p.) & $22.94 \pm 7.18 *$ & $35.42 \pm 7.78 * \circ$ & $46.55 \pm 11.94^{*} \circ$ \\
50 (con p.p.) & $31.59 \pm 7.32 *$ & $68.05 \pm 9.98 * \circ$ & $80.14 \pm 12.4^{*} \circ$ \\
\hline
\end{tabular}

\section{p.p. pompa peristaltica}

$*=p<0.01$ verso valori basali

${ }^{\circ}=p<0.01$ verso valori senza pompa peristaltica 

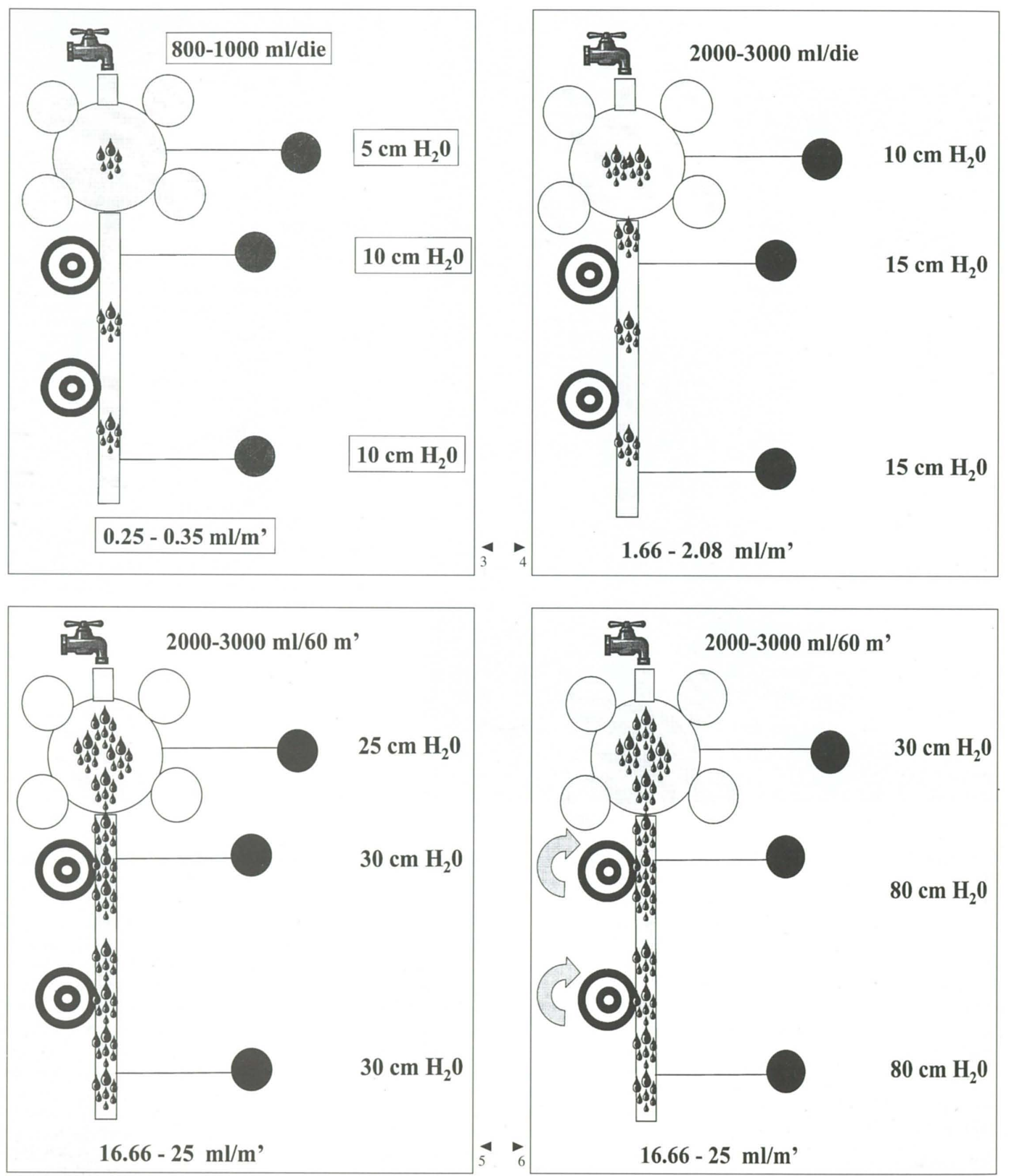

Fig. 3-6 - Flussi e pressioni intracavitarie con introduzione nel modello di quantità di liquidi simulanti l'assunzione di fluidi dai pazienti calcolotici in vari archi di tempo. Pressioni intracavitarie e flussi elevati si ottengono solo introducendo notevoli quantità di liquidi in tempi brevi. 


\section{Modello fisico per lo studio della funzione della terapia idropinica nella calcolosi urinaria}

\section{Materiali e metodi di sperimentazione}

Onde cercare di capire se le leggi fisiche e chimiche sopracitate inerenti l'acqua influiscano sugli effetti della terapia idropinica nelle vie urinarie dell'uomo, abbiamo cercato di costruire un modello sperimentale che ricrei in maniera più possibile aderente alla realtà l'apparato urinario escretore umano.

A tale scopo ci siamo fatti costruire in gomma morbida (Fig. 1) delle sfere cave del diametro di circa $6 \mathrm{~cm}$ che rappresenterebbero le pelvi; all'altezza della circonferenza sono state aggiunte quattro sfere più piccole del diametro di $2.5 \mathrm{~cm}$ comunicanti con la sfera principale e distanziate regolarmente una dall'altra: queste ultime rappresenterebbero i calici. In mezzo a due di queste è stato inserito un tubo dello stesso materiale di $7 \mathrm{~mm}$ di cavità interna sempre collegato alla sfera principale che fungerebbe da uretere. Esattamente alla parte opposta esiste un'altra apertura ad imbuto. Il tutto è come raffigurato nella Figura 1, ma ci viene fornito sezionato esattamente nella metà sagittale (Fig. 2) onde poter agevolmente preparare la parte interna: prima di ciascun esperimento le due parti vengono saldate con colla acrilica e i modelli vengono scartati alla fine di ogni esperimento.

Una volta saldate le due parti il modello viene montato su un supporto in legno e tenuto in sito dalle apparecchiature necessarie alla sperimentazione e precisamente:

1) connessione della parte alta della sfera per il collegamento a pompa da infusione;

2) tre manometri di Claude per la misurazione della pressione interna: uno al livello della "pelvi" uno dell" "uretere prossimale" ed uno di quello "distale";

3) due pompe peristaltiche posizionate una al terzo prossimale e l'altra

\section{TABLE II - STUDIO DELLA POSSIBILE AZIONE "SOLVENTE" DI ALCUNE ACQUE NEI RIGUARDI DELLA MATRICE ORGANICA CHE MANTIENE ADESI I CALCOLI URINARI ALLE VIE ESCRETRICI}

Distacco dei calcoli dal bacinetto: 10 esperimenti per ogni tipo di acqua e di colla con un flusso di $22 \mathrm{ml} / \mathrm{min}$

\begin{tabular}{lccc} 
& Acqua 1 & Acqua 2 & Acqua 3 \\
Colla 1 & 2 & 1 & 3 \\
Colla 2 & 1 & 3 & 4 \\
Colla 3 & 0 & 0 & 1 \\
\hline Acqua 1 Ac. Siena & & Colla 1 Colla di fibrina & \\
Acqua 2 Ac. Vivo & & Colla 2 Salda d'amido & \\
Acqua 3 F. Rocchetta & & Colla 3 Collagene & \\
& &
\end{tabular}

\section{TABLE III - STESSO STUDIO DELLA TABELLA PRECEDENTE, MA CON} L'AGGIUNTA DI “RENELLA” IN UNO DEI CALICI INFERIORI

Distacco dei calcoli dal bacinetto: 10 esperimenti per ogni tipo di acqua e di colla con un flusso di $22 \mathrm{ml} / \mathrm{min}$ con renella in un calice

\begin{tabular}{lccc} 
& Acqua 1 & Acqua 2 & Acqua 3 \\
\hline Colla 1 & 2 & 3 & 6 \\
Colla 2 & 3 & 4 & 7 \\
Colla 3 & 0 & 1 & 4 \\
\hline Acqua 1 Ac. Siena & & Colla 1 Colla di fibrina & \\
Acqua 2 Ac. Vivo & & Colla 2 Salda d'amido & \\
Acqua 3 F. Rocchetta & & Colla 3 Collagene &
\end{tabular}

al terzo distale dell" "uretere": i rotori delle due pompe sono stati rivestiti di morbida gommapiuma e appoggiati all" "uretere" tanto da accluderlo solo per due terzi (circa $5 \mathrm{~mm}$ ) e non completamente con lo scopo di mimare il più realisticamente possibile la funzione peristaltica senza provocare tutte le conseguenze di una pompa rotatoria che funziona all'occlusione del tubo. Allorché funzionanti le pompe hanno sempre lavorato ad un numero costante di giri (30 giri/min).

Per quanto riguarda la renella è stata utilizzata sabbia di mare tenuta a lungo in acqua distillata.

Calcoli renali sono stati recuperati da emissioni spontanee di pazienti con calcolosi o durante interventi chirurgici di calcolosi renale: sono stati utilizzati solo calcoli di ossalato di calcio onde omogeneizzare l'esperimento. I calcoli, se necessario, venivano ridotti a peso variabile fra i 600 ed i
$700 \mathrm{mg}$ e di diametro tra i 3 ed i 4 millimetri.

Abbiamo inoltre utilizzato il nostro modello per verificare l'effetto delle acque sulle matrici che tengono adesi i calcoli renali alla mucosa delle vie escretrici.

Allo scopo abbiamo utilizzato colle adesive biologiche e precisamente:

1) colla di fibrina

2) salda d'amido

3) colla di pesce (collagene), addizionata di piccole quantità di gluteraldeide.

La prima parte dello studio e stata realizzata effettuando serie di 10 esperimenti per ciascun flusso di acqua introdotto nel modello sia con pompe simulanti la peristalsi ferme che in azione. Sono stati utilizzati flussi (attraverso il flussimetro )di $0.55 \mathrm{ml} / \mathrm{min}, 0.7 \mathrm{ml} / \mathrm{min}, 1.38$ $\mathrm{ml} / \mathrm{min}, 2.08 \mathrm{ml} / \mathrm{min}, 33 \mathrm{ml} / \mathrm{min}, 50$ $\mathrm{ml} / \mathrm{min}$ che corrispondono rispettivamente a $800 \mathrm{ml} / 24 \mathrm{~h}, 1000 \mathrm{ml} / 24 \mathrm{~h}$, 
$2000 \mathrm{ml} / 24 \mathrm{~h}, 2000 \mathrm{ml} / 60 \mathrm{~min}$ e 3000 $\mathrm{ml} / 60 \mathrm{~min}$; sono stati registrati sia i flussi in uscita che le pressioni all'interno dei vari segmenti del sistema.

Le stesse serie di esperimenti sono state poi ripetute introducendo su uno dei "calici inferiori" 2 grammi di rena di mare e poi facendo aderire un calcolo di 600-700 mg alla "pelvi" con i tre tipi di colla sopramenzionati. In questa ultima serie di esperimenti sono stati utilizzati tre tipi di acque: una di durezza molto bassa, una di durezza lieve e l'ultima di durezza discreta, provenienti due da acquedotti urbani e l'altra da una fonte minerale.

Più precisamente le acque utilizzate per l'ultima serie di esperimenti sono state:

1) acqua dell'acquedotto di Siena (GF 49.8, Ca $144 \mathrm{mg} / \mathrm{L}$ )

2) acqua dell' acquedotto del Vivo (Amiata) (CF 2.3, Ca $6.2 \mathrm{mg} / \mathrm{L}$ )

3 ) acqua minerale Rocchetta (Gualdo Tadino) (GF 15.4. Ca $55.1 \mathrm{mg} / \mathrm{L}$ ).

I dati ottenuti sono stati elaborati statisticamente utilizzando i Cross-Tabs e Chi-Square test.

\section{Risultati}

Gli esperimenti tendenti a verificare i presupposti teorici dell'effetto washout (Figg. 3-6) hanno confermato, come era del resto logico supporre, che solo con introduzione di notevole quantità di liquido in poche ore si ottengono flussi apprezzabili: infatti introducendo ad esempio $3000 \mathrm{ml}$ in $60 \mathrm{~min}$ si ottiene nel modello un flusso, esattamente come previsto, di 50 $\mathrm{ml} / \mathrm{min}$ : se il modello fosse doppio, come avviene nell'uomo allorché si introducono notevoli quantità di liquido, otterremo un flusso di 25 $\mathrm{ml} / \mathrm{min}$ per ciascun "rene" che è un flusso apprezzabile. Molto interessante è invece il comportamento delle pressioni intracavitarie che partendo da $5 \mathrm{~cm}$ di $\mathrm{H}_{2} \mathrm{O}$ in una "pelvi" con un flusso di $0.25 \mathrm{ml} / \mathrm{min}$ e senza "peristalsi" arrivano a $80 \mathrm{~cm} \mathrm{H}_{2} \mathrm{O}$ con flussi di $25 \mathrm{ml} / \mathrm{min}$ con le pompe peristaltiche in funzione (Tab. I).

La renella posta nel "calice" inferiore viene rimossa totalmente solo in pre- senza di flussi superiori a $20 \mathrm{ml} / \mathrm{min}$ e con pompe peristaltiche in funzione. I calcoli liberi nella pelvi (non adesi alla mucosa) vengono rimossi in una percentuale del $30 \%$ con flussi di 25 $\mathrm{ml} / \mathrm{min}$ e con pompe peristaltiche in funzione: se però è contemporaneamente presente renella nel solito "calice" allora l'espulsione del calcolo avviene nell' $80 \%$ dei casi $(\mathrm{p}<0.01)$. Il comportamento dei calcoli adesi alla pelvi con le tre colle biologiche utilizzate, in presenza delle tre acque in sperimentazione è riportato nelle Tabelle II e III. I calcoli adesi alla pelvi si staccano più facilmente $\mathrm{e}$ quindi vengono eliminati (a flussi di $25 \mathrm{ml} / \mathrm{min}$ e con pompe peristaltiche in funzione) utilizzando l'acqua minerale Rocchetta rispetto alle altre due acque prese in studio: l'analisi statistica non ha mostrato in questo caso una significatività, ma solo una marcata tendenza.

\section{Conclusioni}

Se cerchiamo di farci un'idea sull'attuale stato dell'arte della terapia idropinica della calcolosi urinaria dobbiamo partire dall'opinione molto diffusa in medicina che la contrazione del volume urinario sia uno dei maggiori fattori di rischio per l'urolitiasi e che il semplice incremento della diuresi delle 24 sia di per sé molto efficace nel prevenire 1'incidenza della calcolosi urinaria $(6,9)$. In verità questo concetto è stato supportato per secoli senza dimostrazioni scientifiche corrette: infatti esistono solo pochi studi prospettici sulla terapia idropinica nelle nefrolitiasi, pubblicati su riviste internazionali: per quanto ci risulta sono solo quelli di Frank e De Vries (1966)(16, 19) e quello di Borghi et al (1996)(5). Questo ultimo è riuscito a dimostrare, in uno studio controllato, che pazienti con nefrolitiasi calcica idiopatica possono prevenire la recidiva della calcolosi solo con l'aumento della diuresi senza modificazione della dieta, con l'esclusione dei pazienti ipercalciurici nei quali è indispensabile ridurre il calcio urinario con la dieta o con la tera- pia farmacologica.

Quindi nel 1988 allorché la Consensus Conference che fu pubblicata su JAMA (18) ritenne importante consigliare nei pazienti calcolotici un volume urinario superiore ai 2 litri/die, poteva, dal punto di vista della moderna letteratura internazionale, basarsi solo sul lavoro di Frank e De Vries. Una volta tanto il buon senso ha prevalso sulla fredda razionalità.

Molti Autori d'altra parte hanno evidenziato (anche se in studi non controllati) come particolari condizioni climatiche ed ambientali che provocano disidratazione possano favorire la formazione di calcoli renali. (7, 9, $13,30)$. Fra questi da segnalare il lavoro dello stesso Borghi et al (34) che nel 1993 hanno studiato l'esposizione al rischio litogeno di macchinisti cronicamente esposti ad alte fonti di calore, rilevando che la disidratazione è un grave rischio calcolotico.

Secondo Rose nel $18.6 \%$ di pazienti con calcolosi, la disidratazione deve essere considerata l'agente etiologico (8).

Il basso volume urinario aumenta la concentrazione di tutti i minerali che formano i calcoli e quindi aumenta il rischio della cristalluria. Anche se la concentrazione aumenta logicamente il tasso degli inibitori della cristallizzazione l'aumento della concentrazione dei minerali domina la situazione con aumento proporzionale del rischio.

D'altra parte, anche se non vi sono in letteratura dati recenti, sembra evidente che ci possano essere pochi dubbi sul fatto che un elevato apporto idrico possa ridurre il rischio di infezione urinaria (8) favorendo così, a sua volta, la terapia e la profilassi della calcolosi. Con $2000 \mathrm{ml}$ di diuresi al giorno, un significativo numero di pazienti con calcolosi da ipercalciuria è capace di ridurre sensibilmente il rischio di urolitiasi $(12,13$, $25,41)$.

Secondo alcuni è presupposto fondamentale che la terpia idropinica sia somministrata lungo tutto l'arco della giornata (compresa la notte) e per tutto l'anno (15), ma non tutti sono d'accordo su tale linea (14). Anche se la diuresi del paziente calcolotico 
deve essere abbondante su tutte le 24 ore, è bene ricordare che la fisica dell'acqua che abbiamo prima ricordato ci fa comprendere come la diuresi forzata possa veramente "detergere" le vie urinarie e di conseguenza va evidenziata l'importanza dell'ingestione di grandi quantità di acqua anche in tempi brevi.

A questo punto va ricordato che le varie acque hanno palatabilità diversa: quale esempio limite, sarà molto difficile far ingerire ad un paziente due litri di acqua clorata dell'acquedotto cittadino in poche ore, mentre più agevole sarà convincerlo a bere altrettanta acqua minerale.

Per raggiungere la diuresi di $2000 \mathrm{ml}$ al dì è bene ricordare che è indispensabile ingerire almeno 3 litri di liquidi.

Ma quale deve essere la composizione dell'acqua minerale per il trattamento e la prevenzione della calcolosi?

È onesto affermare che la letteratura internazionale non si è mai occupata dell'argomento, se non del tutto recentemente e con pochissimi contributi, la maggior parte italiani (40) mentre una messe di lavori ha riempito riviste nazionali in molti paesi europei fra i quali l'Italia.

Quindi se è abbastanza semplice affermare che la terapia idropinica è indispensabile nella terapia e nella prevenzione della calcolosi renale, assai più arduo è affrontare il problema di che tipo di acqua ci si deve avvalere e se le acque minerali giochino un ruolo importante in questo campo.

Onde cercare di portare un contributo alla comprensione di tale problema, abbiamo cercato di creare un modello sperimentale che simulasse la funzione delle vie urinarie escretrici (pelvi ed ureteri) dove è maggiore la probabilità di formazioni litiasiche allo scopo di verificare se le leggi fisiche e chimiche che regolano la forza del trasporto delle acque siano valide anche nelle vie urinarie di deflusso e se l'adesività del calcolo alla mucosa delle vie urinarie possa essere influenzata dalla terapia idropinica in generale e dalle acque minerali in particolare.

I risultati del nostro modello mostrano che, almeno nella simulazione, $\mathrm{i}$ liquidi che scorrono nelle vie escretrici si attengono alle stesse leggi delle acque che scorrono nei fiumi o nelle tubature rigide. In particolare la forza di trasporto è molto probabile che segua la legge di Hopkins che prevede al raddoppio della portata, sessantaquattro volte l'aumento della forza di trasporto. Inoltre più l'acqua è ricca di detriti più capacità ha di trasporto e quindi di rimozione dei calcoli ed in questo caso il modello ha dato risultati statisticamente significativi.

A nostro parere, tale sperimentazione pone in evidenza l'efficacia dell'ingestione di una grande quantità di liquidi in poco tempo: infatti se si urina un litro di liquidi in un'ora (evento normale in terapia idropinica termale) si aumenta il flusso ureterale di circa 20 volte con notevole attivazione della peristalsi ureterale: secondo la legge di Hopkins la forza di trasporto è aumentata di diverse centinaia di volte.

Se poi le vie urinarie sono ricche di detriti (renella) l'espulsione del calcolo diventerà ancora più probabile.

Interessanti anche $\mathrm{i}$ dati ottenuti dalle prove di espulsione con calcoli adesi alla "pelvi" con varie colle biologiche: l'acqua oligominerale Rocchetta si è dimostrata molto più attiva come solvente di tali colle rispetto all'acqua dell'acquedotto di Siena e di quello del Vivo sull' Amiata.

L'acqua Rocchetta ha una durezza di 15.4 GF che è molto inferiore a quella dell'acquedotto di Siena, ma è superiore a quella dell'acquedotto del Vivo ed il suo tenore calcico è di $55.1 \mathrm{mg} / \mathrm{L}$ : forse basterebbero questi dati per poter fare intuire le maggiori potenzialità di questa acqua minerale, ma è anche probabile che altre proprietà fisico chimiche di alcune acque minerali al momento non ancora conosciute giochino ruoli determinanti e le differenzino le une dalle altre.

L'attività solvente delle acque è a mio parere un nuovo aspetto importante del problema della calcolosi renale che andrà approfondito ulteriormente anche nell'uomo, ma in ogni caso possiamo già trarre alcune considerazioni conclusive. La prima è che il nostro modello ci ha permesso di evidenziare l'importanza del classico "colpo d'acqua" nella terapia idropinica della calcolosi renale: se è importante infatti un apporto giornaliero elevato di liquidi per inibire la cristallizzazione, l'emissione in tempi brevi di notevole quantità di urina è indispensabile a creare flussi ureterali capaci di attivare la forza di trasporto e di corrosione indispensabile per la rimozione di calcoli all'interno delle vie urinarie.

In secondo luogo paradossalmente hanno più probabilità di espellere calcoli pazienti che eliminino renella per un notevole potenziamento della forza di trasporto.

Infine in questa sperimentazione l'acqua oligominerale Rocchetta si è dimostrata dotata di un maggior potere solvente verso la matrice che tiene adesi i calcoli alla parete della mucosa delle vie urinarie rispetto ad altre acque di acquedotto. 


\section{BIBLIOGRAFIA}

1. Davis SK, Day JA. L'acqua. Torino: Ed. Einaudi, 1961; 110.

2. Monkhouse FJ. Principles of Physical Geography. Londra: Hoddler e Stoughton Ed. 1975; 142-3.

3. Strahler AN. Geografia Fisica. Piccin Ed. Padova, 1984; 389-90.

4. Ross JA, Kirkland PES. Behaviour of the human ureter in health and disease. Churchill Livigstone Ed., Edimburgo, 1972; 24-32.

5. Borghi L, Meschi T, Amato F, Briganti A, Novarini A. Urinary volume, water and recurrences in idiopathic calcium nephrolithiasis: a 5year randomized prospective study. J Urol 1996; 155: 839-43.

6. Ljunghall S, Fellstrom B, Johansson G. Prevention of renal stones by a high fluid intake? Eur Urol 1988; 14: 381-5.

7. Ackermann D, Baumann JM, Futterlieb A, Zingg EJ. Influence of calcium content in mineral water on chemistry and cristallization conditions in urine of calcium stone formers. European Urology 1988; 14: 305-8.

8. Rose A. The medical menagement of renal stone disease. In: Cameron S. et al eds. Oxford Textbook of Clinical Nephrology. Ed. Oxford Med Pub 1992; 1853.

9. Pak C. Urolithiasis, In: Schrier RW, Gottschalk eds. Diseases of the Kidney. $5^{\circ}$ Ed. Little, Brown and C. ed, Boston, 1993; 25.

10. Hrusca KA, Seltzer JR,Grieff M. Nephrolithiasis. In: Schrier RW e Gottschalk CW eds. Diseases of the Kidney. $6^{\circ}$ Ed.,Little, Brown and C. Ed., 1996; 752.

11. Smith BL. The pathogenesis and medical treatment of urolithiasis. Seminars in Nephrology 1990; 10: 31-52.

12. Minetti L, Colussi G, Surian M. Nefrolitiasi (fisiopatologia e clinica) Acta Med. Ed., Roma, $1985 ; 45$.

13. Micali F, Porena M, Vespasiani G. La calcolosi urinaria. Wellcome ed. Roma, 1979; 104.

14. Frank M, Vries A. Prevention of urolithiasis. Archives of environmental Health 1966; 13: 625-30.

15. Consensus Conference: Prevention and Treatment of kidney stones. JAMA 1988; 260: 977-81.

16. Frank M, De Vries A, Atsmon A, Lazebnik J, Kochwa S. Epidemiological investigation of urolithiasis in Israel. J Urol 1959; 81: 482-97.

17. Ulmann A, Sayegh F, Clavel J, Lacour B. Frequences des recidives lithiasiques apres une cure de diurese simple ou associée a un traitment par un diuretique ou le phosphore. La Presse Med 1984; 13: 1257-60.

18. Elliot JP, Gordon JO, Evans JW, Platt L. A stone season. A 10 years retrospective study of 768 surgical stone cases with respect to seasonal variation. J Urol 1975; 4, 114 : 574-7.

19. Borghi L, Meschi T, Amato F, Novarini A, Romanelli A, Cigala F. Hot occupation and nephrolithiasis. J Urol 1993; 150: 1757-60.

20. Albertazzi A, Palmieri PF, Spisni C, Di Guglielmo R, Polidoro MM. Changes in the water-elctrolyte balance after administration of oligomineral water in nephrolithiasis patients. Clin Ter 1985; 115 : 181-92.

21. Aroldi A, Graziani G, Passerini $\mathrm{P}$, et al. High fluid intake or pharmacological therapy in recurrent stone former patients? Proc Eur Dial Trans Eur Ren Ass 1985; 21: 743-6. 